\title{
Correction to: A Connection Between Sports and Matroids: How Many Teams Can We Beat?
}

\author{
Ildikó Schlotter ${ }^{1}$ (D) Katarína Cechlárová
}

Received: 26 January 2017 / Accepted: 18 September 2017 / Published online: 4 October 2017

(C) Springer Science+Business Media, LLC 2017

\section{Correction to: Algorithmica DOI 10.1007/s00453-016-0256-2}

\section{Background}

Our article "A connection between sports and matroids: How many teams can we beat?" [4] deals with a problem we called MinStanding $(S)$. The motivation behind this problem comes from the following situation. Given an ongoing sports competition among a set $T$ of teams, with each team having a current score and some matches left to be played, we ask whether it is possible for our distinguished team $t \in T$ to obtain a final standing with at most $r$ teams finishing before $t$.

For a general model that can be applied to various sports competitions, we denoted by $S$ the set of all possible outcomes of a match, where each outcome is a pair $\left(p_{1}, p_{2}\right)$ of non-negative reals corresponding to the situation where the match ends with the home team obtaining $p_{1}$ points and the away team obtaining $p_{2}$ points. If $S$ contains pairs $(\alpha, 0)$ and $(0, \beta)$ for some positive $\alpha$ and $\beta$, then we say that $S$ is well based.

Given a set $S$ of outcomes with $|S|=k+1$, the above question boils down to the following graph labelling problem:

The online version of the original article can be found under doi:10.1007/s00453-016-0256-2.

Ildikó Schlotter

ildi@cs.bme.hu

Katarína Cechlárová

katarina.cechlarova@upjs.sk

1 Budapest University of Technology and Economics, Budapest 1521, Hungary

2 Institute of Mathematics, Faculty of Science, P.J. Šafárik University, Jesenná 5, 04001 Košice, Slovakia 


\section{$\operatorname{MinSTANDING}(S)$ :}

Instance: A triple $(G, c, r)$ where $G=(V, A)$ is a directed multigraph, $c: V \rightarrow$ $\mathbb{R}$ describes vertex capacities, and $r$ is an integer.

Question: Does there exist an assignment $p: A \rightarrow\{0, \ldots, k\}$ such that the number of vertices in $V$ violating the inequality

$$
\sum_{a=(v, u) \in A} \alpha_{p(a)}+\sum_{a=(u, v) \in A} \beta_{p(a)} \leq c(v)
$$

is at most $r$ ?

\section{The Error and its Correction}

In Theorem 3 of our article [4], we incorrectly stated that "MinStanding( $S)$ is W[1]hard with parameter $|V(G)|-r$ for any well-based set $S$ of outcomes, even if the (undirected version of the) input graph $G$ is claw-free".

The presented (erroneous) proof gave an FPT reduction from the W[1]-hard INDEPENDENT SET problem. Although the reduction itself is correct, we erroneously claimed that INDEPENDENT SET is W[1]-hard on claw-free graphs. However, this is not true, since INDEPENDENT SET can be solved in polynomial time on claw-free graphs [2,3]. What holds true is that INDEPENDENT SET is W[1]-hard on $K_{1,4}$-free graphs, as proved by Hermelin, Mnich, and Van Leeuwen [1]. So the term "claw-free" in the above statement (Theorem 3 of our article [4]) should be replaced by " $K_{1,4}$-free".

The correct statement of the theorem is thus the following.

Theorem 1 MinStanding $(S)$ is W[1]-hard with parameter $|V(G)|-r$ for any wellbased set $S$ of outcomes, even if the (undirected version of the) input graph $G$ is $K_{1,4}$-free.

Proof We give a simple FPT reduction from the W[1]-hard INDEPENDENT SET problem, which is known to be W[1]-hard even on $K_{1,4}$-free graphs [1]. Let $G$ be the input graph and $\ell$ the parameter given. The constructed instance of $\operatorname{MinSTANDING}(S)$ will be $(\vec{G}, c,|V(G)|-\ell)$ where $\vec{G}$ is an arbitrarily oriented version of $G$, and $c$ is the constant zero function.

Now, it is easy to see that a set $X$ of vertices in $G$ is independent if and only if there is a score assignment on $\vec{G}$ in which vertices of $X$ are not violating. Note that here we make use of the fact that $S$ is well based.

Acknowledgements We are grateful to Matthias Mnich who kindly pointed out our error.

\section{References}

1. Hermelin, D., van Leeuwen, E.J.: Parameterized complexity of induced graph matching on claw-free graphs. Algorithmica 70(3), 513-530 (2014)

2. Minty, G.J.: On maximal independent sets of vertices in claw-free graphs. J. Comb. Theory Ser. B 28(3), 284-304 (1980) 
3. Sbihi, N.: Algorithme de recherche d'un stable de cardinalité maximum dans un graphe sans étoile. Discrete Math. 29(1), 53-76 (1980)

4. Schlotter, I., Cechlárová, K.: A connection between sports and matroids: How many teams can we beat? Algorithmica (to appear). doi:10.1007/s00453-016-0256-2 